\title{
Pseudomediation: A reply and more data'
}

BRUCE EARHARD ${ }^{2}$ AND GEORGE MANDLER ${ }^{3}$

UNIVERSITY OF TORONTO

\begin{abstract}
The experimental critique by Jenkins \& Foss of the pseudomediation argument is considered. Their mediation data apparently support the pseudomediation argument and their data on unlearning fail to differentiate response and associative learning. Two additional experiments are presented which show (a) the expected unlearning during Stage II of the mediated chaining paradigm, and (b) evidence for facilitation in the mediated inference paradigm.
\end{abstract}

\section{Problem}

Jenkins \& Foss (1965) have questioned the reasoning and the empirical data presented in our previous study (Mandler \& Earhard, 1964). This is surprising as a comparison of their Fig. 1 with our Fig. 1 shows a parallel course of events in the three stages of both studies. The failure of some of their dependent variables to show the pseudomediation effect would appear to result from the lack of equivalence between experimental and control conditions which is evident in the Stage I learning data of their study. In addition, Jenkins \& Foss' intervening recall test and their $8 \mathrm{sec}$. intertrial interval provide Ss with time to consider the task involved, and could lead to the application of mediational rules which would work against the pseudomediation effect (Earhard \& Mandler, 1965). Given that our main finding was reproduced, it is puzzling that Jenkins \& Foss fail to mention that this finding is contrary to mediation theory which must predict interference rather than facilitation in Stage III.

Jenkins \& Foss are correct in suggesting that there should be differential forgetting and unlearning between experimental and control conditions during Stage II. Their recall data suggest that this is not the case, but their method of testing recall does not provide a suitable test of the unlearning of associations which constitutes the basis of our arguments.

The distinction between response recall and the associative phase of paired associate learning applies also to studies of unlearning and forgetting (cf. McGovern, 1964). The finding of Jenkins \& Foss that response recall did not differ for experimental and control groups does not necessarily mean the forgetting of Stage I associations is also equal. McGovern's results show quite clearly that the loss of associative connections is not necessarily deducible from response recall data.

In the first experiment reported below, unlearning of Stage I associations during Stage II learning will be investigated by testing for associative connections rather than response recall. As in the McGovern study, Ss are provided with Stage I stimulus and response terms and asked to pair them correctly after Stage II learning.

The second experiment examines the problem of mediated interference. In this paradigm, Stage I consists of an A-B list, Stage II of a B-C list and Stage III of an $\mathrm{A}-\mathrm{Cr}$ list in which $\mathrm{A}$ and $\mathrm{C}$ terms are randomly paired. Mediation theory predicts that Stage III learning in this paradigm will be inferior to that of a control condition (A-B, D-C, A-Cr). As Jenkins (1965) has noted, our analysis of mediation paradigms suggests that Stage III of the experimental condition should be learned more easily than that of the control condition because of the greater forgetting of interfering items in the experimental group as compared with the control group.

\section{Method}

In Experiment 1, the availability of Stage I associations was tested following Stage II learning. Three paradigms were used. For all paradigms Ss learned a common A-B list in Stage I, In Stage II, Ss learned a B-C list in Paradigm 1, a C-A list in Paradigm 2, and a C-D list in Paradigm 3. It was expected that little or no unlearning of Stage I associations would occur in Paradigm 3. Unlearning was expected in Paradigm 1 and 2 with the most unlearning expected in Paradigm 1 (cf. Earhard \& Mandler, 1965).

The items used were the same as those used by Mandler \& Earhard (1964), with the addition of the words "phial" and "cameo". Three different pairings were used for each paradigm and five different list orders were used. The presentation rate was 2:2 with no special intertrial interval and the interstage interval was only long enough for instructions to be administered. Twelve Ss per paradigm learned each list to a criterion of one perfect trial. Following the learning of Stage I and Stage II lists, Ss were given a sheet of paper on which were listed the A items from Stage $I$ in random order, and a separate sheet, on which the $B$ items were listed in a random order. Ss were asked to write the appropriate B item next to each A item.

Experiment 2 repeated the Mandler \& Earhard(1964) study exactly as far as list construction, presentation, and instructions were concerned. After learning the A-B and B-C lists, the experimental Ss were given an A-Cr list so constructed that no pair of A-Cr terms had been linked with the same B term in either Stage I or II. The control group learned an A-B, D-C, A-Cr 


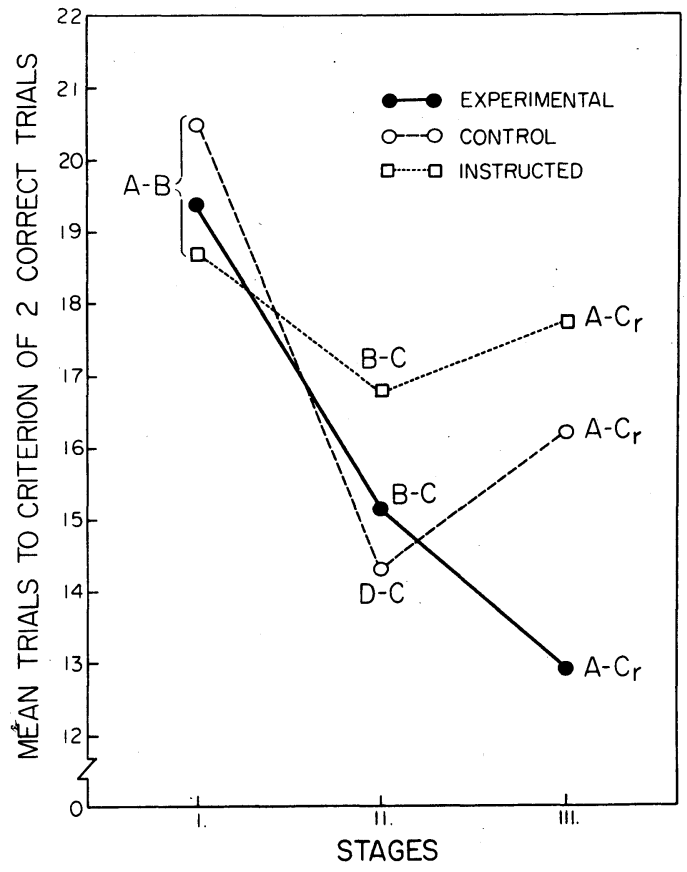

Fig. 1. Mean trials to two correct trials for all three groups of Experiment 2 in the three stages.

sequence of lists. Twenty-four Ss were used in each group. An additional $10 \mathrm{Ss}$ were run in an Instructed condition. This condition was identical to the experimental condition above, except that Ss were told specifically about the mediation hypothesis, and were given examples of mediated chains that were expected to develop in Stages I and II. They were then given the randomly-paired Stage III list. If Ss used the mediation rule, mediated interference should develop in Stage III and should override the pseudomediation faciliation effect.

\section{Results}

The matching test in Experiment 1 showed the following mean number of Stage I associations recalled per S:

Paradigm 1: A-B, B-C $\quad 5.3$

Paradigm 2: A-B, C-A 6.8

Paradigm 3: A-B, C-D $\quad 8.0$

In Paradigm 3 all Ss correctly paired all eight pairs from Stage I. The other two paradigms were ordered in the expected fashion. The lack of variance in Paradigm 3 made a t-test impossible, but a t-test between Paradigms 1 and 2 resulted in a $t$ of 2.20 , $\mathrm{p}<.05$. When Ss in all three conditions were divided into those who made no errors and those who made one error or more, Fisher's exact test yielded a significant difference between Paradigm 3 and both Paradigm 1 $(p<.01)$ and Paradigm $2(p<.05)$. The three conditions were well matched on performance on Stage I; the mean trials to a criterion of one correct trial were 13.0, 14.5 and 14.0 for Paradigms 1, 2, and 3 respectively.
For Experiment 2, the number of correct responses during the first six trials and mean number of trials to the criterion of two correct trials were analyzed. The former failed to show a significant difference between the experimental and control conditions; but when the latter variable was considered, the difference between experimental and control conditions, which is shown in Fig. 1, was significant $(t=2.1, p<.05)$. The Instructed condition showed the anticipated impairment but was not significantly different from the control condition. It was, however, significantly different from the experimental condition $(t=2.5, p<.02)$. None of the differences in Stage I were significant.

\section{Diseussion}

The data of Experiment 1 clearly show the expected degree of unlearning of associative connections assumed by Mandler \& Earhard (1964). When response recall is controlled, as it was not in the Jenkins \& Foss study, the data are consistent with the reasoning that leads to the prediction of pseudomediation. Stage I A-B associations are weakened when the second stage includes either of the two members of the first stage.

Experiment 2 has demonstrated a significant effect for the A-Cr paradigm with laboratory established associations. That effect is facilitative, i.e., exactly opposite to that predicted by the verbal mediation hypothesis, and consistent with our analysis of mediation paradigms. The impaired performance that resulted under the Instructed condition is consonant with our position that mediational effects are dependent upon the application of rules or organizing principles (cf. Earhard \& Mandler, 1965). If a rule is provided, as in the case of the present experiment, or if material is selected in accordance with overlearned rules (e.g., associative or semantic norms), or even if matching techniques are employed in the test list to reduce task complexity and memory load (e.g., Schulz, Weaver \& Ginsberg, 1965), mediational effects, as opposed to interference and forgetting effects, become apparent in performance.

\section{References}

Earhard, B., \& Mandler, G. Mediated associations: Paradigms, controls, and mechanisms. Unpublished manuscript, 1965.

Jenkins, J. J. Comments on pseudomediation. Psychon. Sci., 1965, 2, 97-98.

Jenkins, J. J., \& Foss, D. J. An experimental analysis of pseudomediation. Psychon. Sci., 1965, 2, 99-100.

Mandler, G., \& Earhard, B. Pseudomediation: Is chaining an artifact? Psychon. Sci., 1964, 1, 247-248.

McGovern, Jean B. Extinction of associations in four transfer paradigms. Psychol. Monogr., 1964, 78, No. 16 (Whole No. 593).

Schulz, R. W., Weaver, G. E., \& Ginsberg, S. Mediation with pseudomediation controlled: Chaining is not an artifact! Psychon. Sci., 1965, 2, 169-170.

Notes

1. This research was supported by Grant GB-810 from the National Science Foundation and by Grant APA-64 from the National Research Council, Canada. We are most grateful to Sherry R. Anderson, George E. McCullough and Shirley Osler for experimental assistance.

2. Now at Dalhousie University, Halifax, N. S.

3. Now at University of California, San Diego, La Jolla, California. 